\title{
Endoscopic Management of Staple Line Leak after Bariatric Surgery: Surgeon's Perspective
}

\author{
Yoona Chung, Dae Geun Park and Yong Jin Kim \\ Bariatric and Metabolic Surgery Center, $\mathrm{H}+$ Yangji Hospital, Seoul, Korea
}

Laparoscopic sleeve gastrectomy (LSG) has become a standalone primary procedure as a bariatric metabolic surgery since the early 2000s. The overall complication rate of LSG is reported to range from $2 \%$ to $15 \%$. Staple line leakage (SLL) remains a major adverse event and occurs in approximately $1-6 \%$ of patients. Choosing the optimal treatment modality is a complex process. Clinicians must understand that nutritional support and drainage of fluid collection are essential for initial management. Conservative endoscopic management and sufficient drainage can resolve approximately 70\% of SLLs. Endoscopic management of bariatric complications has been rapidly evolving in recent years and can be considered in all patients who are hemodynamically stable. We will review the available endoscopic management techniques, including stent placement (self-expanding stents and bariatric-specific stents), clipping, tissue sealant application, and internal drainage (double-pigtail stents [DPS] placement, endoscopic vacuum therapy, and septotomy). Stent placement remains the mainstream treatment for SLLs. However, healing with stents requires multiple sessions/ stents and a long course of recovery. Endoscopic internal drainage is gaining popularity and has the potential to be a superior method. The importance of early intervention and combined endoscopic methods should be recognized. Clin Endosc 2021;54:805809

Key Words: Drainage; Morbid; Obesity; Stents; Surgical stapling

\section{INTRODUCTION}

Laparoscopic sleeve gastrectomy (LSG) has become a standalone primary procedure as a bariatric metabolic surgery since the early 2000s. ${ }^{1}$ Initially, it had been introduced as a first-step procedure to biliopancreatic diversion or duodenal switch in morbidly obese patients. Over time, it has gained popularity due to the relatively less demanding surgical technique, preservation of the natural anatomy, and a lower complication rate than that of other precedent procedures such as Roux-en-Y gastric bypass (RYGB). ${ }^{2}$ The overall complication rate of LSG

Received: November 13, 2020 Revised: January 18, 2021

Accepted: January 20, 2021

Correspondence: Yong Jin Kim

Bariatric and Metabolic Surgery Center, H+ Yangji Hospital, 1636, Nambusunhwan-ro, Gwanak-gu, Seoul 08779, Korea

Tel: +82-10-5382-6758, Fax: +82-2-879-1610, E-mail: yjgs1997@gmail.com

ORCID: https://orcid.org/0000-0003-1222-2121

(c) This is an Open Access article distributed under the terms of the Creative Commons Attribution Non-Commercial License (http://creativecommons.org/ licenses/by-nc/3.0) which permits unrestricted non-commercial use, distribution, and reproduction in any medium, provided the original work is properly cited. is reported to range from $2 \%$ to $15 \% .^{3}$ Complications include leakage, bleeding, and stricture.

Among all complications, staple line leakage (SLL) remains a major adverse event with increased morbidity and mortality. ${ }^{4}$ SLL occurs in approximately 1-6\% of patients and can develop into chronic fistulas. Most SLLs (approximately $85 \%$ of all cases) occur in the gastroesophageal junction. ${ }^{3}$ The cause of SLLs can be either mechanical or ischemic. In both scenarios, the intraluminal pressure of the sleeve exceeds the tissue and staple line resistance leading to dehiscence of the staple line. It is generally believed that leaks presenting within the first 2 days of surgery are caused by mechanical causes (distal stenosis, tension along the staple line, tissue trauma, and hematoma) and technical failure in choosing the optimal staple height for the tissue thickness. Meanwhile, leaks caused by ischemia present $>5-6$ days after surgery. ${ }^{5}$ Rosenthal et al. ${ }^{6}$ categorized SLLs into acute (within 7 days), early (within 1-6 weeks), late (after 6 weeks), and chronic (after 12 weeks). The management of SLLs differs according to the time of diagnosis from the time of surgery, which will be further discussed below.

The diagnosis of SLLs can be difficult. Tachycardia with 
a heart rate of $>120$ bpm may be the only clinical presentation in many cases. Gastrointestinal series or computed tomography (CT) may assist in the diagnosis but can produce false-negative results, and must not delay return to the operating room (the intention of the authors was that the performance of the CT scan itself should not delay the decision for emergency operation) if the patient is hemodynamically unstable.

Choosing the optimal treatment modality for a patient with SLL, whether it be surgery or endoscopic management, is a complex process. However, clinicians must understand that the use of broad-spectrum antibiotics, proton pump inhibitors, nutritional support, and draingae of fluid collection are all essential for initial management. ${ }^{8}$ The surgical options for chronic and refractory leak after LSG include conversion to RYGB, total or proximal gastrectomy (which may entail an esophagojejunostomy), or a Roux-en-Y fistulojejunostomy. However, reoperation has a high rate of morbidity and mortality in the setting of active intra-abdominal contamination. ${ }^{9}$ Conservative endoscopic management and sufficient drainage can resolve approximately $70 \%$ of SLLs. ${ }^{10}$ Endoscopic management of bariatric complications has been rapidly evolving in recent years and can be considered in all patients who are hemodynamically stable. We will review the available endoscopic management techniques, including stent placement (self-expanding stents and bariatric-specific stents), clipping, tissue sealant application, and internal drainage (double-pigtail stents [DPS] placement, endoscopic vacuum therapy [EVT], and septotomy).

\section{STENT PLACEMENT}

Endoscopic stenting is a minimally invasive procedure that allows early ambulation, enteral feeding, and greater patient comfort. ${ }^{11}$ Fully covered self-expanding metal stents (SEMSs) are preferred for the management of SLLs. SEMSs have been the subject of a few meta-analyses in recent years. The success rate has been improving, and the most recent meta-analysis reported a success rate of $92 \% .^{10}$ The most common and frustrating complication is stent migration. The migration rate can range from 23\% to 59\%. ${ }^{10,12}$ Migration requires repeated procedures for repositioning, and migration into the small bowel necessitates surgical intervention. Other common complications include nausea, vomiting, reflux, and discomfort, which may lead to intolerance.

Recently, bariatric-specific stents have been introduced into the market. Some examples include the MEGA stent (an ultra-sized fully covered stent; Taewoong Medical, Gimpo, Ko- rea), BETA stent (with two migration cuffs; Taewoong Medical), and GASTROSEAL stent (MITECH, Seoul, Korea). ${ }^{12}$ The success rate of the MEGA stent was reported to be $82 \%$. The migration rate was $18 \%$, which is considerably lower than that of standard SEMSs. The number of procedures and stents required per patient was 3 and 1.3, respectively. ${ }^{13}$ These numbers are significantly lower than those for standard SEMSs. However, pain, vomiting, and excessive salivation were observed in all patients. Patient education and assurance are important for preventing intolerance and improving outcomes.

A few additional points need to be addressed. The endoscopist must remember that drainage, absence of distal obstruction, and destruction of any epithelization of the fistula tract must be achieved along with the main procedure. Oral intake is routinely withheld for $24-48 \mathrm{~h}$ for full stent expansion, and a liquid to semi-solid diet can be tolerated for the duration of stenting. Plain radiography can be performed every 1-2 weeks to confirm the stent location. Any change in symptoms (pain intensity, fever, or vomiting) or recurrence of symptoms should be addressed, as this may suggest stent migration. ${ }^{11}$ The duration of stent dwelling varies greatly. However, most authors recommend 6-8 weeks. ${ }^{10}$ Earlier intervention, SLLs $<10$ $\mathrm{mm}$, no history of previous gastric banding, and first endoscopy were all reported to be associated with better success of endoscopic treatment. ${ }^{14}$ After the optimal 8 weeks of stenting, an endoscopic evaluation should be performed to assess the healing state. Gastrografin injection into the presumed leakage site is performed after the removal of the current stent. The endoscopist should be prepared for immediate reinsertion of another stent in cases of dye leakage during endoscopy. If there is evidence of leakage, the next endoscopy can be considered 4 weeks after reinsertion of another stent (the removed stent cannot be reinserted and the authors wanted to stress this point).

\section{CLIPPING}

The over-the-scope-clip (OTSC; Ovesco Endoscopy, Tübingen, Germany) system is a relatively novel clipping system that has been reported to successfully treat gastric leaks and fistulas. ${ }^{13}$ It can be applied for the treatment of SLLs $<10 \mathrm{~mm} .{ }^{15} \mathrm{~A}$ systematic review published in 2017 reported a success rate of $86.3 \% .{ }^{16}$ However, this study included cases with additional or concomitant endoscopic procedures. In a recent meta-analysis, the success rate of clipping alone was $67 \%$. This further highlights the importance of combined endoscopic methods. ${ }^{10}$ Considering the low success rate of the OTSC system alone in SLLs and its cost, a paper published in 2020 concluded that 
the OTSC system should be considered an option only for patients who could not tolerate other endoscopic treatments. ${ }^{17}$ The OTSC system is also inappropriate for chronic fistulas with difficult-to-approximate fibrotic tissue. ${ }^{18}$ Complications related to clipping (anchor migration, tear, etc.) are rare. ${ }^{10}$

\section{TISSUE SEALANT APPLICATION}

Tissue sealants include fibrin glue and cyanoacrylate. Fibrin glue is the most commonly used sealant for fistula closure, and its application after various operations, including LSG, biliopancreatic diversion, and total gastrectomy, has been reported. ${ }^{19}$ In addition to occluding the defect itself, fibrin glue also plays a role in wound healing by inducing cellular response to tissue damage and promoting neovascularization and fibroblast proliferation by forming matrix-building strands. ${ }^{20}$ The current success rate ranges from $92.8 \%$ to $100 \%,{ }^{10}$ although most cases require repeated sessions for complete closure. The main factors for failure are a large orifice or non-compliance of the patient to the scheduled application. The main advantages of fibrin glue are its easy application, low cost, and lack of severe complications. Brushing with a cytology brush has been recommended to clean debris and granulation tissue from the fistula orifice before sealant application. ${ }^{21}$ Overall, complications related to fibrin glue are rare. In one study, $12.5 \%$ patients presented with pain and fever after fibrin glue injection. ${ }^{21}$

Cyanoacrylate is a synthetic glue that functions as a mechanical sealant with high adhesive and antibacterial properties. It is six times less expensive than fibrin glue. Its efficacy has been demonstrated to be as high as $96.8 \%$ in various anastomotic leakages. ${ }^{22}$ However, its application has been limited to date because of its difficult handling and delivery, as well as potential proinflammatory effects. ${ }^{10}$

\section{INTERNAL DRAINAGE}

Achieving adequate drainage in the treatment of SLLs is paramount to success. When proper drainage is not initially performed, SLLs may recur and develop into chronic fistulas even with a long duration of treatment with other endoscopic modalities such as SEMS placement. DPS placement, EVT, and septotomy are discussed below in more detail.

\section{Double-pigtail stents placement}

The DPS is a useful option for the drainage of collected fluids into the sleeved stomach. This induces the collapse of the abscess cavity, leading to closure of the SLL. ${ }^{12}$ The drain itself induces reepithelialization and guides the healing of the fistula. ${ }^{23}$ The reported success rate ranges from $79 \%$ to $95 \%$ when coupled with enteral nutrition. ${ }^{12,24}$ The mean time to heal was reported to be 55.5 days, with 2.95 procedures required per patient. ${ }^{24}$ This method should be applied to SLLs with a fistula orifice $<10 \mathrm{~mm}$ and can be utilized with SEMSs in larger leaks. ${ }^{25}$ Any external drains should immediately be removed once internal drainage is achieved to prevent the formation of an enterocutaneous fistula. ${ }^{12} \mathrm{~A}$ complication rate of $13.7 \%$ has been reported, with the most severe complication being migration of DPS, which can lead to splenic parenchymal hemorrhage or abscess. ${ }^{26}$ Most reports recommend at least 4 weeks of nasojejunal feeding. The most significant advantages of DPS are the minimal discomfort experienced by patients and the high success rate even in chronic SLLs.

\section{Endoscopic vacuum therapy}

EVT is becoming an increasingly popular tool for the management of SLLs. This is because of the ability of endoscopic intraluminal EVT to successfully maintain drainage of fluid collection, which is not possible with SEMSs. ${ }^{9}$ The success rate was reported to be $89 \%$ in patients with SLL in whom drainage with SEMS failed. ${ }^{27}$ EVT can be performed under conscious sedation or general anesthesia. Injection of botulinum toxin into the pylorus has been suggested as an adjunct therapy to reduce distal pressure and ensure better healing. Cases of stricture and hemorrhage of adjacent vascular structures in patients with erosive conditions have been reported as EVT-related complications. ${ }^{28}$ Compared with EVT, DPS drainage requires the placement of two stents and carries the risk of migration with the subsequent complications mentioned above. However, it is relatively less invasive and does not require potential general anesthesia. Conversely, EVT can offer a considerably shorter treatment period than DPS placement because it induces healing without the formation of a chronic fistula.

\section{Septotomy}

Septotomy first appeared in the literature as stricturotomy for chronic fistulas after RYGB. This procedure enables internal drainage while allowing deviation of oral intake into the sleeved stomach. ${ }^{29}$ The success rate was reported to be $80 \%$ in a small case series in 2020 and $100 \%$ in another case series in 2017. ${ }^{18,30}$ Argon plasma and needle knife as a cutting device have been used in previous reports, although any device with hemostatic technology can be applied. Septotomy requires multiple sessions and, accordingly, has also been called "progressive" endoscopic septotomy. ${ }^{30}$ The entire cavity should be gradually exposed with each session by completely cutting the 


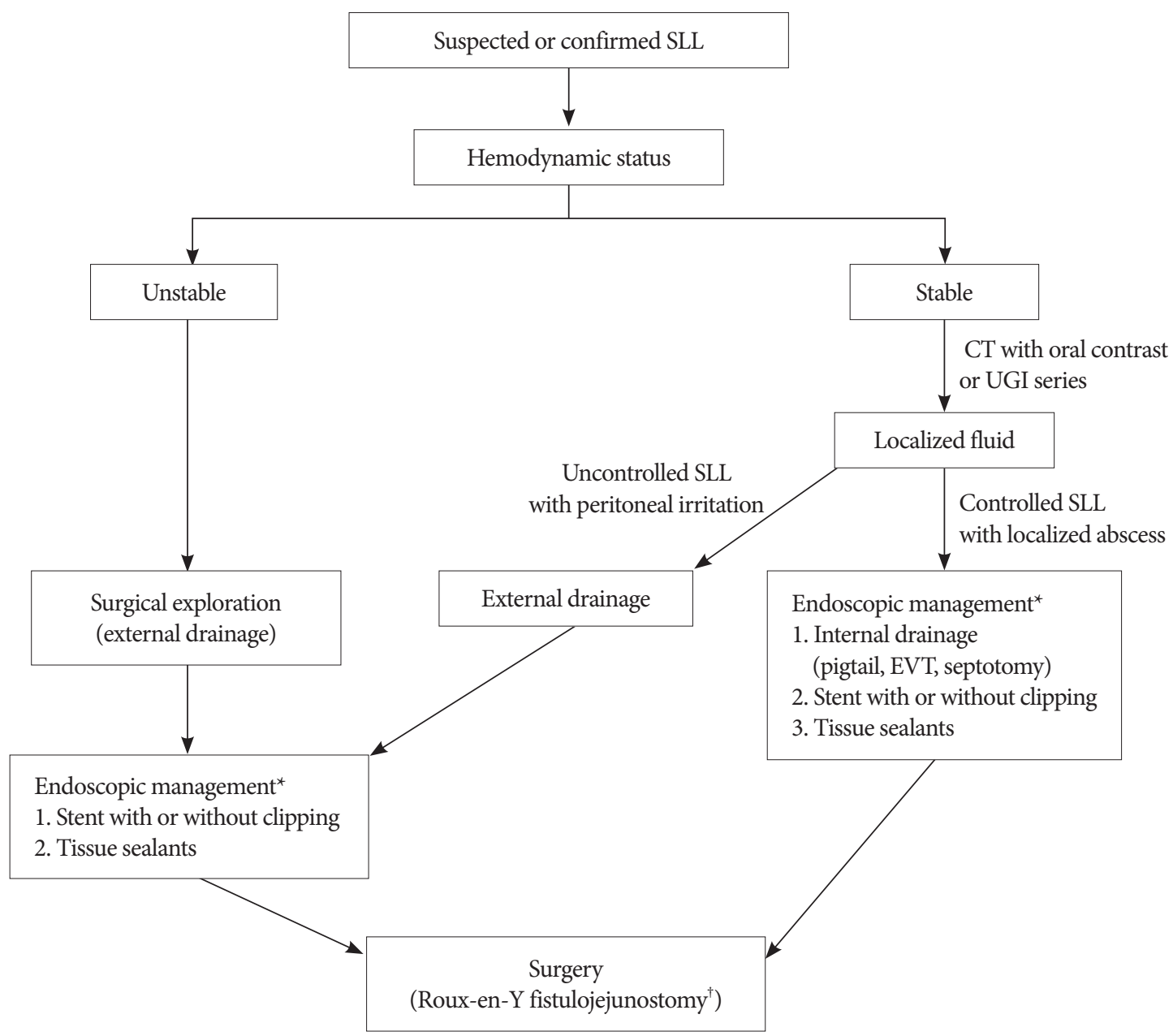

Fig. 1. Management algorithm for staple line leakage. CT, computed tomography; EVT, endoscopic vacuum therapy; SSL, staple line leakage; UGI, upper gastrointestinal. "The procedures are recommended in the order of the item numbers. The method of internal drainage should be selected according to the experience and preference of the endoscopist. ${ }^{\dagger}$ Roux-en-Y fistulojejunostomy is the preferred surgical method.

residual septum. The number of sessions required for healing was reported to range from 1.8 to 5 sessions per patient. ${ }^{18}$

\section{CONCLUSION}

Stent placement remains the mainstream treatment for SLLs. However, healing with stents requires multiple sessions/ stents and a long course of recovery. A management algorithm (Fig. 1) is provided as a reference for clinicians. Endoscopic internal drainage is gaining popularity and has the potential to be a superior method. The importance of early intervention and combined endoscopic methods should be recognized.
Conflicts of Interest

The authors have no potential conflicts of interest.

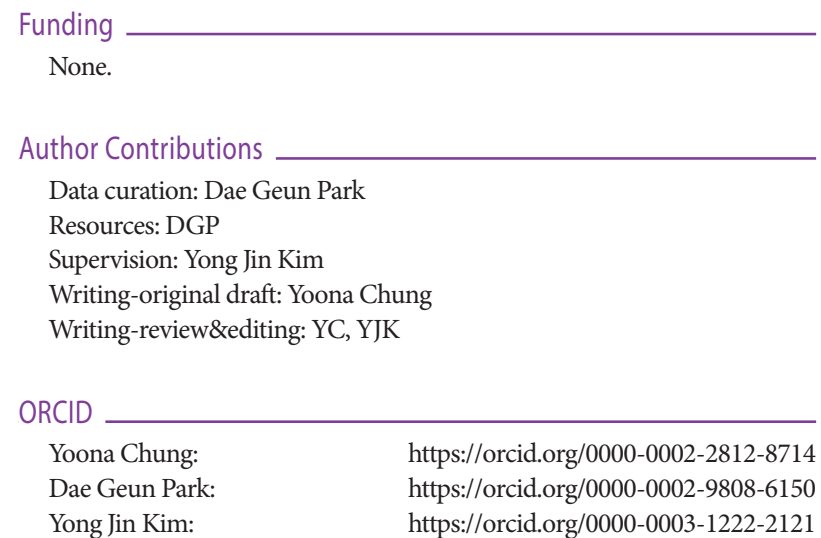




\section{REFERENCES}

1. Gagner M, Deitel M, Erickson AL, Crosby RD. Survey on laparoscopic sleeve gastrectomy (LSG) at the Fourth International Consensus Summit on Sleeve Gastrectomy. Obes Surg 2013;23:2013-2017.

2. Fridman A, Moon R, Cozacov Y, et al. Procedure-related morbidity in bariatric surgery: a retrospective short- and mid-term follow-up of a single institution of the American College of Surgeons Bariatric Surgery Centers of Excellence. J Am Coll Surg 2013;217:614-620.

3. Moon RC, Shah N, Teixeira AF, Jawad MA. Management of staple line leaks following sleeve gastrectomy. Surg Obes Relat Dis 2015;11:54-59.

4. Ma IT, Madura JA. Gastrointestinal complications after bariatric surgery. Gastroenterol Hepatol (NY) 2015;11:526-535.

5. Márquez MF, Ayza MF, Lozano RB, Morales M del MR, Díez JMG, Poujoulet RB. Gastric leak after laparoscopic sleeve gastrectomy. Obes Surg 2010;20:1306-1311.

6. Rosenthal RJ, International Sleeve Gastrectomy Expert Panel, Diaz AA, et al. International Sleeve Gastrectomy Expert Panel Consensus Statement: best practice guidelines based on experience of $>12,000$ cases. Surg Obes Relat Dis 2012;8:8-19.

7. Walsh C, Karmali S. Endoscopic management of bariatric complications: a review and update. World J Gastrointest Endosc 2015;7:518-523.

8. Okazaki O, Bernardo WM, Brunaldi VO, et al. Efficacy and safety of stents in the treatment of fistula after bariatric surgery: a systematic review and meta-analysis. Obes Surg 2018;28:1788-1796.

9. Ward MA, Ebrahim A, Clothier JS, et al. Factors that promote successful endoscopic management of laparoscopic sleeve gastrectomy leaks. Surg Endosc 2021;35:4638-4643.

10. Rogalski P, Swidnicka-Siergiejko A, Wasielica-Berger J, et al. Endoscopic management of leaks and fistulas after bariatric surgery: a systematic review and meta-analysis. Surg Endosc 2021;35:1067-1087.

11. Hamed H, Said M, Elghadban H, Elgeidie A. Outcome and adverse events of endoscopic bariatric stents for management of leakage after bariatric surgery. Obes Surg 2020;30:982-991.

12. Shehab H. Enteral stents in the management of post-bariatric surgery leaks. Surg Obes Relat Dis 2018;14:393-403.

13. Shehab H, Abdallah E, Gawdat K, Elattar I. Large bariatric-specific stents and over-the-scope clips in the management of post-bariatric surgery leaks. Obes Surg 2018;28:15-24.

14. Christophorou D, Valats J-C, Funakoshi N, et al. Endoscopic treatment of fistula after sleeve gastrectomy: results of a multicenter retrospective study. Endoscopy 2015;47:988-996.

15. Nedelcu M, Manos T, Cotirlet A, Noel P, Gagner M. Outcome of leaks after sleeve gastrectomy based on a new algorithm adressing leak size and gastric stenosis. Obes Surg 2015;25:559-563.

16. Shoar S, Poliakin L, Khorgami Z, et al. Efficacy and safety of the overthe-scope clip (OTSC) system in the management of leak and fistula after laparoscopic sleeve gastrectomy: a systematic review. Obes Surg 2017;27:2410-2418.

17. Mizrahi I, Grinbaum R, Elazary R, et al. Staple line leaks following laparoscopic sleeve gastrectomy: low efficacy of the over-the-scope clip. Obes Surg 2021;31:813-819.

18. Diaz R, Welsh LK, Perez JE, et al. Endoscopic septotomy as a treatment for leaks after sleeve gastrectomy: meeting presentations. Digestive Disease Week 2019. Endosc Int Open 2020;8:E70-E75.

19. Choi YY, Cho JY, Kim YJ. Successful endoscopic management of an esophagojejunostomy leak using fibrin glue injection after a total gastrectomy. Am Surg 2011;77:376-378.

20. Bonanomi G, Prince JM, McSteen F, Schauer PR, Hamad GG. Sealing effect of fibrin glue on the healing of gastrointestinal anastomoses: implications for the endoscopic treatment of leaks. Surg Endosc 2004;18:1620-1624.

21. Assalia A, Ilivitzki A, Ofer A, et al. Management of gastric fistula complicating laparoscopic sleeve gastrectomy with biological glue in a combined percutaneous and endoscopic approach. Surg Obes Relat Dis 2018;14:1093-1098.

22. Kotzampassi K, Eleftheriadis E. Tissue sealants in endoscopic applications for anastomotic leakage during a 25 -year period. Surgery 2015;157:79-86.

23. Lorenzo D, Guilbaud T, Gonzalez JM, et al. Endoscopic treatment of fistulas after sleeve gastrectomy: a comparison of internal drainage versus closure. Gastrointest Endosc 2018;87:429-437.

24. Donatelli G, Ferretti S, Vergeau BM, et al. Endoscopic Internal Drainage with Enteral Nutrition (EDEN) for treatment of leaks following sleeve gastrectomy. Obes Surg 2014;24:1400-1407.

25. Southwell T, Lim TH, Ogra R. Endoscopic therapy for treatment of staple line leaks post-laparoscopic sleeve gastrectomy (LSG): experience from a large bariatric surgery centre in New Zealand. Obes Surg 2016;26:1155-1162.

26. Giuliani A, Romano L, Marchese M, et al. Gastric leak after laparoscopic sleeve gastrectomy: management with endoscopic double pigtail drainage. A systematic review. Surg Obes Relat Dis 2019;15:1414-1419.

27. Leeds SG, Burdick JS. Management of gastric leaks after sleeve gastrectomy with endoluminal vacuum (E-Vac) therapy. Surg Obes Relat Dis 2016;12:1278-1285.

28. Archid R, Wichmann D, Klingert W, et al. Endoscopic vacuum therapy for staple line leaks after sleeve gastrectomy. Obes Surg 2020;30:13101315.

29. Campos JM, Ferreira FC, Teixeira AF, et al. Septotomy and balloon dilation to treat chronic leak after sleeve gastrectomy: technical principles. Obes Surg 2016;26:1992-1993.

30. Shnell M, Gluck N, Abu-Abeid S, Santo E, Fishman S. Use of endoscopic septotomy for the treatment of late staple-line leaks after laparoscopic sleeve gastrectomy. Endoscopy 2017;49:59-63. 